\title{
INTERACTION OF MUON BEAM WITH PLASMA DEVELOPED DURING IONIZATION COOLING *
}

\author{
S. Ahmed $^{1 \dagger}$, D.M. Kaplan ${ }^{2}$, T.J. Roberts ${ }^{3}$, L.K. Spentzouris ${ }^{2}$, K.Beard ${ }^{3}$, \\ ${ }^{1}$ Thomas Jefferson National Accelerator Facility, Newport News, VA 23606, USA \\ ${ }^{2}$ Illinois Institute of Technology, Chicago, IL 60616, USA \\ ${ }^{3}$ Muons, Inc., Batavia, IL 60510, USA
}

\section{Abstract}

Particle-in-cell simulations involving the interaction of muon beam (peak density $10^{18} \mathrm{~m}^{3}$ ) with Li plasma (ionized medium) of density $10^{16}-10^{22} \mathrm{~m}^{-3}$ have been performed. This study aimed to understand the effects of plasma on an incoming beam in order to explore scenario developed during the process of ionization cooling. The computer code takes into account the self-consistent electromagnetic effects of the beam interacting with plasma. This study shows that the beam can pass through the plasma of densities four order of magnitude higher than the beam peak density. The low density $\left(10^{16}\right)$ plasma is completely displaced by the beam, however, the strong wake is observed for similar densities of beam and plasma. Study reveals the signature of plasma wakefield acceleration.

\section{INTRODUCTION}

There are several emerging technologies in particle accelerators for example, international linear collider (ILC), compact linear collider (CLIC), plasma/dielectric wakefield accelerators (PWFA/DWA) etc. Muon collider is a compact machine (diameter $\sim 2 \mathrm{Km}$ ) which has been envisioned as a future lepton circular collider for exploring the secret of universe. This machine has unique feature of significantly low synchrotron radiation and hence smaller energy spread because the muon is heavier than electron $\left(\mathrm{m}_{\mu}=207 \mathrm{~m}_{e}\right)$. To achieve high luminosity $\left(L=2 \times 10^{34}\right.$ $\mathrm{cm}^{-2} \mathrm{~s}^{-1}$ ) beam size at the interaction point (IP) should be small $\left(L \propto 1 / \sigma^{2}\right)$, which for the muon beam is achieved by ionization cooling [1]. In this process, beam is passed through a low $\mathrm{Z}$ material (minimum multiple scattering) where it looses its momentum; the longitudinal component $\left(p_{\|}\right)$is regained by accelerating the beam by a radio frequency (rf) cavity - see Fig. 1. The change in normalized emittance is obtained as a net result of heating due to multiple scattering and cooling. The governing equation is defined as [1]

$$
\frac{d \epsilon_{N}}{d s}=-\frac{1}{\beta^{2}}\left|\frac{d E_{\mu}}{d s}\right| \frac{\epsilon_{N}}{E_{\mu}}+\frac{\beta_{\perp}(0.014 G e V)^{2}}{2 \beta^{3} E_{\mu} m_{\mu} X_{0}}
$$

In this cooling mechanism, ionization is created by the beam and the density of ionization increases gradually with

\footnotetext{
* Authored by Jefferson Science Associates, LLC under U.S. DOE Contract No. DE-AC05-06OR23177. The U.S. Government retains a non-exclusive,paid-up, irrevocable, world-wide license to publish or reproduce this manuscript for U.S. Government purposes. †sahmed@jlab.org
}

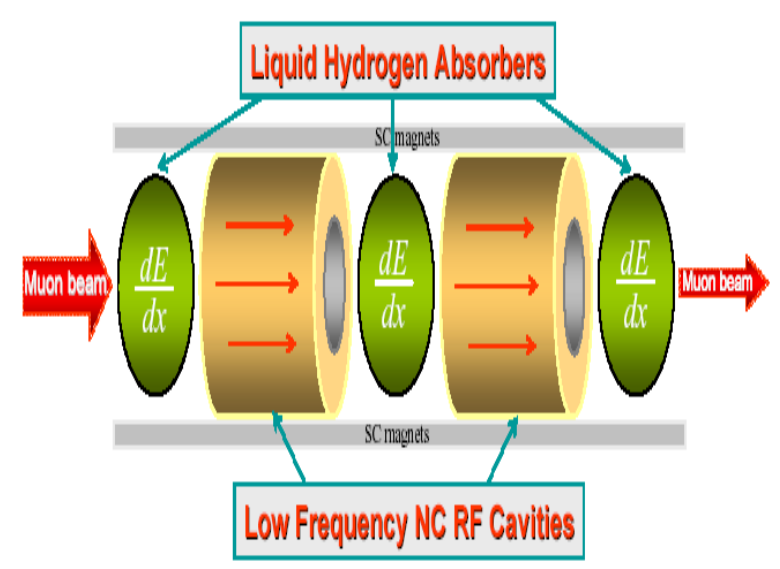

Figure 1: Schematic of ionization cooling for muon beam.

subsequent bunches passing through the medium. It is therefore important to understand the collective effects as a result of beam interaction with plasma. A realistic model should take into account the complete process, the evolution of ionization and its interaction with incoming beams. However, modeling such phenomena at very high pressure (above 760 torr) is complex and needs fluid code to start with the initial ionization, followed by the particle-in-cell (PIC) simulations for moderately high plasma density.

To study the collective effects involving the interaction of beam with densely ionized medium, it is justified to assume that plasma has been formed by several bunches passing through the absorber. This model can address the following questions.

1. How does the electron concentration of an absorbing material ( $\mathrm{Li}$ absorber) interact with the incoming $\mu$ beam?

2. The excitation of plasma wave and wakefields for $\mu^{-}$ and $\mu^{+}$beams propagating inside plasma.

3. The dependence of the amplitude of a plasma wake on different plasma densities.

4. The effects of external magnetic field on the suppression of magnitude of wakefield.

\section{MODEL DESCRIPTION}

We have performed particle-in-cell simulations of the processes involving the interaction of beam with plasma 
Table 1: Parameter of study

\begin{tabular}{lcc}
\hline Parameters & Symbol (Unit) & Value \\
\hline Bunch profile & - & Gaussian \\
Particles & - & $\mu^{-}, \mu^{+}$ \\
Bunch Population & $N_{b}$ & $1 \times 10^{12}$ \\
Bunch radius & $r_{b}(\mathrm{~mm})$ & 3 \\
Bunch Length & $L_{b}(\mathrm{~mm})$ & 40 \\
Reference momentum & $\mathrm{P}(\mathrm{MeV} / \mathrm{c})$ & 200 \\
Rest mass of muon & $m_{\mu}\left(\mathrm{MeV} / \mathrm{c}^{2}\right)$ & 105.7 \\
Relativistic beam factor & $\gamma_{b}$ & 2.1 \\
Relativistic velocity factor & $\beta_{b}$ & 0.88 \\
Pulse length & $\tau_{p}(\mathrm{ps})$ & 133.34 \\
Total Energy & $E_{t o t}(\mathrm{MeV})$ & 226 \\
Total Charge & $Q_{t o t}(\mathrm{nC})$ & 160 \\
Peak beam density & $n_{b}\left(\mathrm{~m}^{-3}\right)$ & $10^{18}$ \\
Plasma frequency of beam & $\omega_{b}(\mathrm{rad} / \mathrm{s})$ & $10^{9}$ \\
Plasma & - & $\mathrm{Li}$ \\
\hline
\end{tabular}

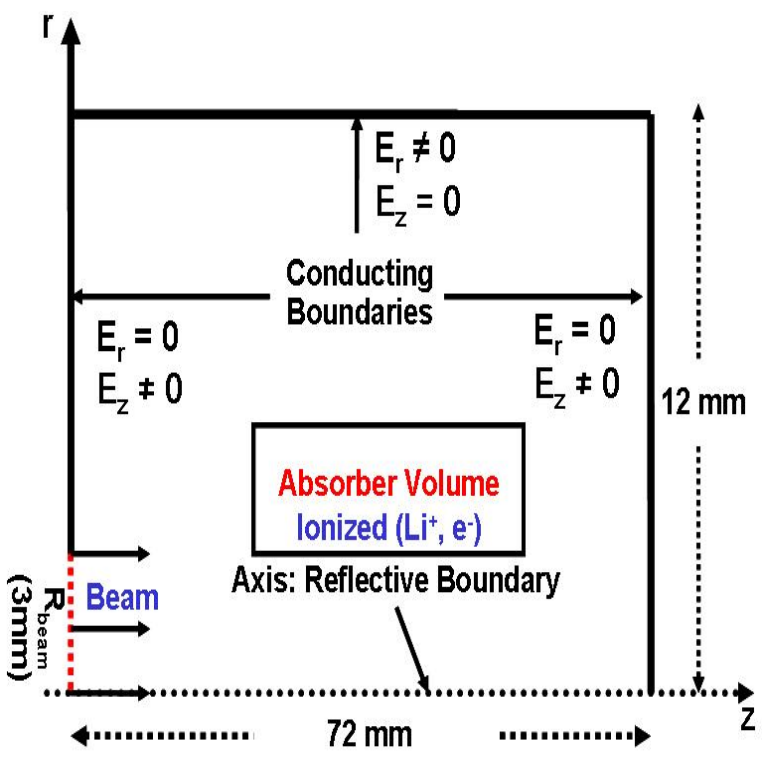

Figure 2: Setup of computational model.

using XOOPIC, a two dimensional self-consistent electromagnetic PIC code based on the finite-difference timedomain (FDTD) method [2]. The parameters chosen for this study are summarized in Table 1 . The setup of the computational model is shown in Fig. 2. The conducting walls satisfy the electromagnetic boundary conditions (i.e. tangential components of E-field are zero), however, the symmetric $z$-axis behaves as a reflective boundary which means the particles hitting the $z$-axis are reflected back into the simulation region. For the best use of computational resources, the size of simulation region considered in this study is $72 \mathrm{~mm} \times 12 \mathrm{~mm}$. At time $t=0$, beam starts traveling into the cold Li plasma; ions are massive hence modeled as stationary uniform background and electrons are uniformly distributed with zero initial velocity. The interaction of charged particle's beam with plasma excites plasma wave. The frequency of oscillation for charge density $n$, mass $m$ and charge $q$ of a particle in plasma is defined as

$$
f_{p}=\frac{1}{2 \pi} \sqrt{\frac{n q^{2}}{\epsilon_{0} m}}
$$

For example, the density $n_{p}=1 \times 10^{18} \mathrm{~m}^{-3}$ corresponds to the plasma frequency $f_{p} \sim 9 \mathrm{GHz}$.
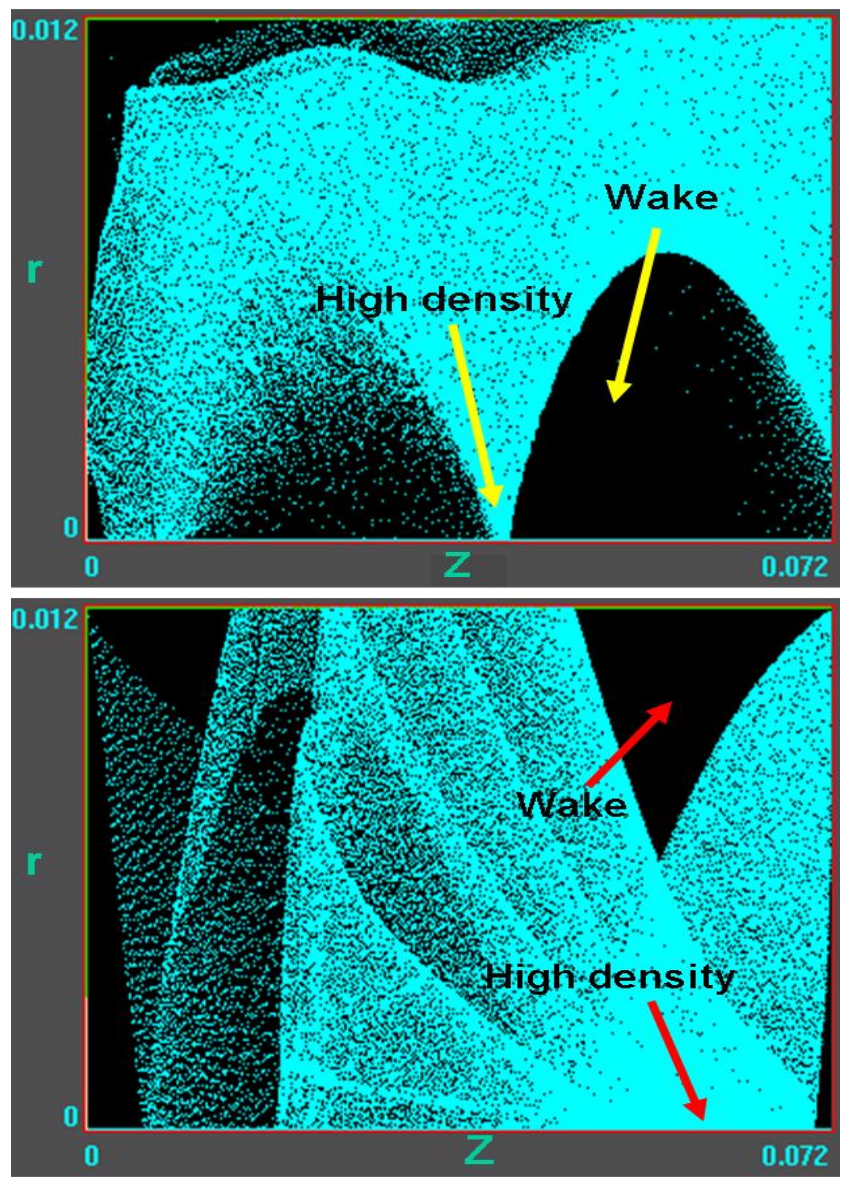

Figure 3: Top and bottom graphs correspond to the evolution of $e^{-}$-plasma due to the interaction of $\mu^{-}$and $\mu^{+}$beams at a time snapshot of $t=300$ ps during which the bunch head moves to a distance of $80 \mathrm{~mm}$.

\section{RESULTS AND DISCUSSION}

We have performed simulations for a range $\left(10^{16}-10^{22}\right.$ $\mathrm{m}^{-3}$ ) of plasma densities and noticed the excitation of strong wakefield and plasma oscillations only in the case when the beam and plasma are of similar densities. For the brevity of the paper, we therefore present the complete picture of matched case - muon beam of density $n_{b}=1 \times 10^{18}$ $\mathrm{m}^{-3}$ interacting with the plasma of density $n_{p}=1 \times 10^{18}$ $\mathrm{m}^{-3}$. Fig. 3 shows a time snapshot involving the interaction of $\mu^{-}$and $\mu^{+}$beams with plasma. The electrons in the plasma are repelled by the $\mu^{-}$-beam and attracted by the 

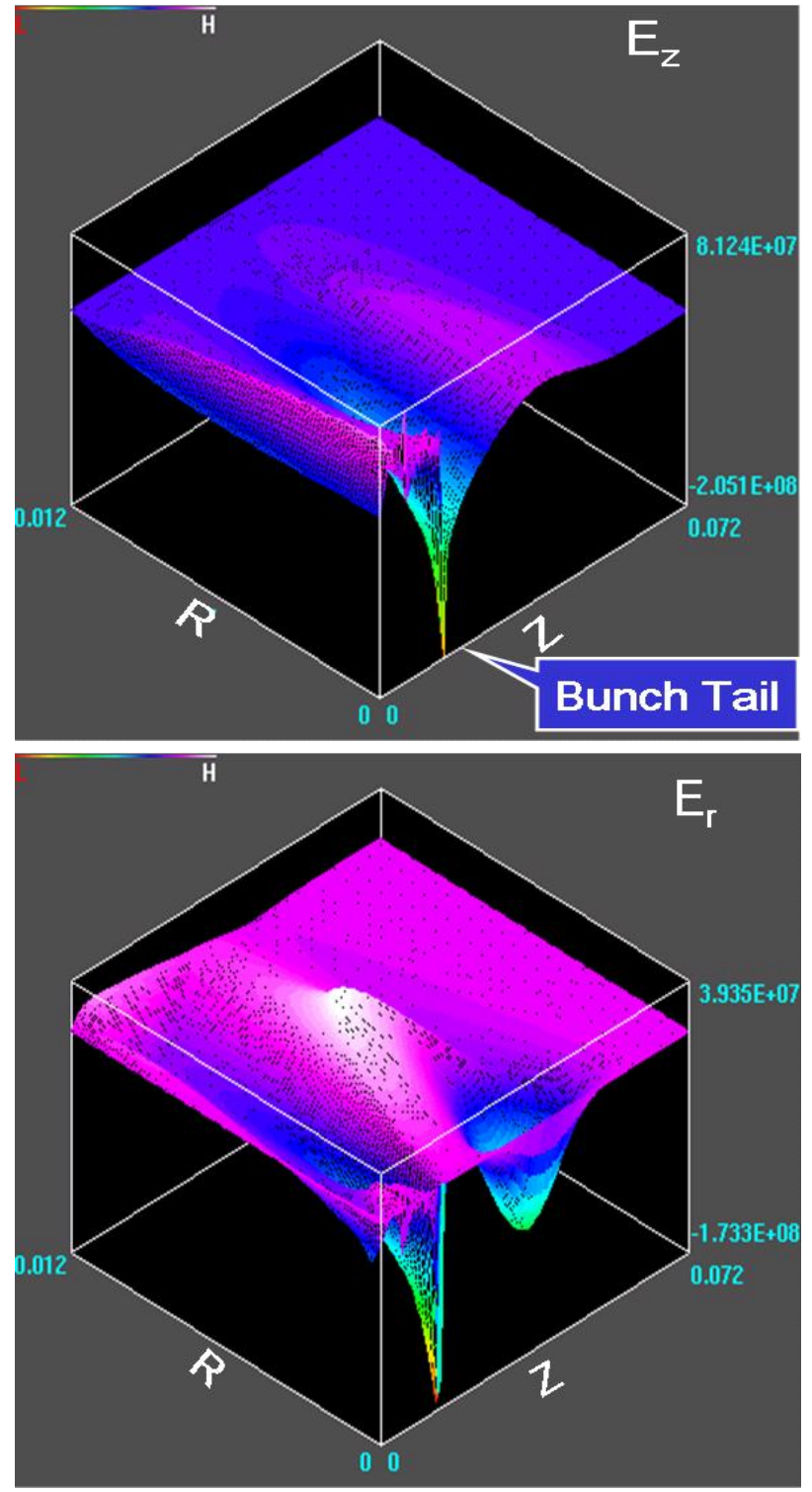

Figure 4: Top and bottom graphs correspond to the distribution of $E_{z}$ and $E_{r}$ in the $r z$-plane at a time snapshot $t=$ 210 ps.

$\mu^{+}$-beam. The repulsion of electrons due to $\mu^{-}$beam results in the absence of electrons which generates positive image charges (wakefield); which in turn pulls the electrons back causing plasma oscillation. It is important to note that head of the beam creates wake, however, the effect is witnessed by the tail. The peak-to-peak distance of the plasma wave is $32 \mathrm{~mm}$ corresponds to $f_{p} \sim 9 \mathrm{GHz}$, which is consistent with the plasma density, $n_{p}=1 \times 10^{18} \mathrm{~m}^{-3}$. The condition where the beam and plasma densities are same results in the resonance causing strong oscillation - see top Fig. 3. In the case of $\mu^{+}$beam interaction with plasma, electrons response is exactly opposite to that of $\mu^{-}$beam - see bottom Fig. 3. The details of the complex phenomena needs further investigation. Fig. 4 shows the distribution of axial and radial field components $E_{z}$ and $E_{r}$ in the $r z$-plane at a time

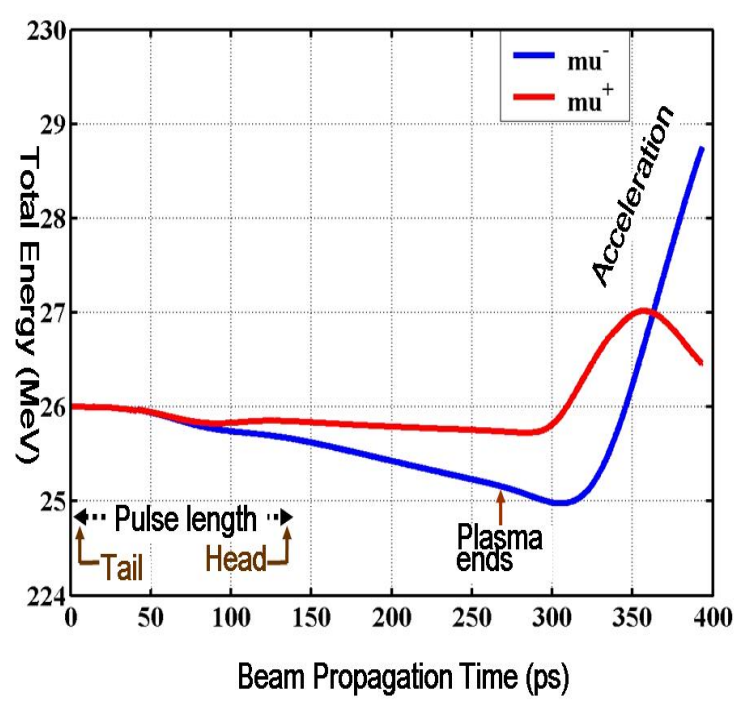

Figure 5: Time evolution of $\mu^{-}$and $\mu^{+}$beam's total energy.

$t=210 \mathrm{ps}$ by which the head of the bunch travels $63 \mathrm{~mm}$. The negatively directed electric fields push the beam in the forward direction as well as compress. Fig. 5 shows the evolution of total energy for $\mu^{-}$and $\mu^{+}$beams while traveling through the plasma. As expected, beam first transfers energy to the plasma $-\mu^{-}$beam loses more energy than $\mu^{+}$. This means that the wakefield is strong in the case of $\mu^{-}$ beam interaction which pushes the tail of the beam forward as soon as beam leaves the plasma. This increase in energy is due to wakefield acceleration.

\section{CONCLUSION}

We have performed particle-in-cell simulations involving the interaction of muon beam with the pre-ionized medium (plasma) developed in the process of muon ionization cooling. This study reveals the excitation of strong wakefield and plasma oscillations for a matched condition - beam density matched to that of plasma, showing resonant behaviour. The magnitude of wakefield due to $\mu^{-}$ beam is stronger than the $\mu^{+}$beam due to the repulsion of plasma electrons. Wake is generated by the head of the bunch, however, the tail witness the effect. There is a gain in beam total energy as a result of plasma wakefield acceleration. Further study requires the investigation of emittance dilution in the process of beam plasma interaction.

\section{REFERENCES}

[1] Charles M. Ankenbrandt et. al., Status of muon collider research and development and future plans, Phys. Rev. ST. Accel. Beams, 2, 081001 (1999).

[2] The Plasma Theory and Simulation Group at the University of California at Berkeley, http://ptsg.eecs.berkeley.edu. 